\title{
openheart Direct and indirect effect of the COVID-19 pandemic on patients with cardiomyopathy
}

Daniel J Hammersley (D) , ${ }^{1,2}$ Rachel J Buchan, ${ }^{1,2,3}$ Amrit S Lota, ${ }^{1,2}$ Lukas Mach, ${ }^{1,2}$ Richard E Jones, ${ }^{1,2}$ Brian P Halliday, ${ }^{1,2}$ Upasana Tayal, ${ }^{1,2}$ Devendra Meena, ${ }^{4}$ Abbas Dehghan, ${ }^{4}$ loanna Tzoulaki, ${ }^{4,5,6}$ A John Baksi, ${ }^{2}$ Antonis Pantazis, ${ }^{2}$ Angharad M Roberts, ${ }^{1,2}$ Sanjay K Prasad, ${ }^{1,2}$ James S Ware ${ }^{1,2,3}$

- Additional supplemental material is published online only. To view, please visit the journal online (http://dx.doi.org/10. 1136/openhrt-2021-001918).

To cite: Hammersley DJ, Buchan RJ, Lota AS, et al. Direct and indirect effect of the COVID-19 pandemic on patients with cardiomyopathy. Open Heart 2022;9:e001918. doi:10.1136/

openhrt-2021-001918

Received 14 December 2021 Accepted 4 January 2022

Check for updates

(c) Author(s) (or their employer(s)) 2022. Re-use permitted under CC BY-NC. No commercial re-use. See rights and permissions. Published by BMJ.

For numbered affiliations see end of article.

Correspondence to Dr James S Ware; j.ware@ imperial.ac.uk

\section{ABSTRACT}

Objectives (1) To evaluate the prevalence and hospitalisation rate of COVID-19 infections among patients with dilated cardiomyopathy (DCM) and hypertrophic cardiomyopathy $(\mathrm{HCM})$ in the Royal Brompton and Harefield Hospital Cardiovascular Research Centre (RBHH CRC) Biobank. (2) To evaluate the indirect impact of the pandemic on patients with cardiomyopathy through the Heart Hive COVID-19 study. (3) To assess the impact of the pandemic on national cardiomyopathy-related hospital admissions.

Methods (1) 1236 patients (703 DCM, $533 \mathrm{HCM}$ ) in the RBHH CRC Biobank were assessed for COVID-19 infections and hospitalisations; (2) 207 subjects (131 cardiomyopathy, 76 without heart disease) in the Heart Hive COVID-19 study completed online surveys evaluating physical health, psychological well-being, and behavioural adaptations during the pandemic and (3) 11447 cardiomyopathy-related hospital admissions across National Health Service (NHS) England were studied from NHS Digital Hospital Episode Statistics over 2019-2020. Results A comparable proportion of patients with cardiomyopathy in the RBHH CRC Biobank had tested positive for COVID-19 compared with the UK population $(1.1 \%$ vs $1.6 \%, p=0.14)$, but a higher proportion of those infected were hospitalised ( $53.8 \%$ vs $16.5 \%, p=0.002)$. In the Heart Hive COVID-19 study, more patients with cardiomyopathy felt their physical health had deteriorated due to the pandemic than subjects without heart disease (32.3\% vs $13.2 \%, p=0.004)$ despite only $4.6 \%$ of the cardiomyopathy cohort reporting COVID-19 symptoms. A $17.9 \%$ year-on-year reduction in national cardiomyopathyrelated hospital admissions was observed in 2020. Conclusion Patients with cardiomyopathy had similar reported rates of testing positive for COVID-19 to the background population, but those with test-proven infection were hospitalised more frequently. Deterioration in physical health amongst patients could not be explained by COVID-19 symptoms, inferring a significant contribution of the indirect consequences of the pandemic. Trial registration number NCT04468256

\section{Key questions}

What is already known about this subject?

- Pre-existing cardiovascular disease is associated with increased mortality for those infected with COVID-19; however, there are limited data available evaluating risk for individual cardiovascular conditions.

- Numerous indirect effects of the COVID-19 pandemic have been reported, including extensive interruptions to emergency and routine care. The extent and impact of these indirect consequences are yet to be characterised in detail.

What does this study add?

- Patients with cardiomyopathy had similar reported rates of testing positive for COVID-19 to the background UK population, but those with test-proven infection were hospitalised more frequently.

- One-third of patients with cardiomyopathy experienced a subjective deterioration in their physical health as a result of the pandemic, in spite of a significantly lower proportion experiencing possible COVID-19 symptoms, inferring a significant contribution of the indirect consequences of the pandemic

- A $17.9 \%$ year-on-year reduction in nationa cardiomyopathy-related hospital admissions was observed in 2020, with the lowest hospitalisation rates occurring during the first UK lockdown.

How might this impact on clinical practice?

- This study provides information on the impact of the COVID-19 pandemic on a specific patient group; studies such as this may be important in guiding future recommendations in the event of further waves of infection.

This study also characterises how the indirect effects of the pandemic, such as interruptions to clinical care, have impacted patients with cardiomyopathy. The insight provided may help to prioritise clinical needs for patients with cardiomyopathy and in turn guide adaptations to clinical services in order to meet these. 


\section{INTRODUCTION}

The true impact of the COVID-19 pandemic on patients with cardiovascular disease is multifaceted and challenging to measure in its entirety. An association has been demonstrated between pre-existing cardiovascular disease and mortality for those infected with COVID19. ${ }^{12}$ However, more granular data is required to evaluate the prevalence of infection and the risk associated with individual cardiovascular conditions, in order to accurately advise patients. It is additionally recognised that the indirect effects of the pandemic, arising from interruptions to emergency and routine care for patients with cardiovascular disease, represent a further source of excess morbidity and mortality, although data detailing the extent of this are currently limited. ${ }^{3}$

The cardiomyopathies represent a heterogeneous group of disorders characterised by structural and functional abnormalities of the myocardium, in the absence of coronary artery disease, hypertension, valvular disease or congenital heart disease sufficient to cause the observed abnormality. ${ }^{4}$ The health needs of patients affected by these conditions are frequently complex and require long-term multi-disciplinary input, rendering such patients vulnerable to interruptions in the delivery of care during the pandemic. We aimed to comprehensively assess both the direct and indirect impact of the pandemic on patients with cardiomyopathy.

\section{METHODS}

To address the outlined unmet clinical needs, we undertook three parallel research initiatives.

Our first initiative evaluated patients with dilated cardiomyopathy (DCM) and hypertrophic cardiomyopathy (HCM) enrolled in the Royal Brompton and Harefield Hospital Cardiovascular Research Centre (RBHH CRC) Biobank. This prospectively recruited cohort comprised consecutive ambulatory patients referred to clinic or for a cardiovascular magnetic resonance (CMR) scan at The Royal Brompton Hospital between 2009 and 2016. The study population thus represents a single-centre cohort of patients referred from a network of over 30 regional hospitals. Cardiovascular diagnosis was confirmed at the time of enrolment using established criteria. ${ }^{56}$ Eligibility for this research initiative included a diagnosis of DCM or HCM and written consent to prospectively gather further clinical follow-up data, including information from electronic registries and National Health Service (NHS) databases. Patients were excluded if they had died prior to January 2020, as this predated the first reported case of COVID-19 in the UK. Primary care records, hospital records and patient questionnaires were collected for patients within this cohort to evaluate the prevalence of COVID-19 infection, COVID-19 hospitalisation and the proportion of patients advised to shield.

During the first lockdown in the UK, the government issued advice to individuals identified as 'clinically extremely vulnerable' to refrain from all unnecessary social contact (ie, to 'shield'). People were centrally identified as 'clinically extremely vulnerable' if they suffered with one of a number of prespecified conditions, or on a case-to-case basis at a discretion of their clinicians. ${ }^{7}$ DCM, HCM and heart failure were not among this list of prespecified conditions. ${ }^{7}$ It was, therefore, anticipated that the majority of shielding recommendations in this cohort had been made at the discretion of their clinicians.

The primary outcome measure was a test-confirmed COVID-19 infection using either a reverse transcriptionPCR test on a respiratory sample or an antibody test on a blood sample; the secondary outcome measures were (1) the proportion of confirmed COVID-19 cases requiring hospitalisation and (2) the proportion of individuals advised to shield. The outcomes were compared with simultaneously published rates of COVID-19 infection, hospitalisation and shielding in the UK population, gathered from the UK Government website. ${ }^{8}$ Patient health data were collected between 20 October 2020 and 2 November 2020. Published COVID-19 data from the UK, including cumulative COVID-19 cases based on positive tests and COVID-19 hospitalisations in the UK population, was accessed up until 2 November 2020.

\section{The Heart Hive COVID-19 study}

Our second initiative utilised the Heart Hive (https:// www.thehearthive.org/), an online portal and registry of patients with self-reported clinically diagnosed cardiomyopathy, and people without heart disease. The Heart Hive portal allows participants to self-enrol and enter their own health data. Research studies are added to the portal and participants are notified if they meet eligibility criteria and invited to enrol. The Heart Hive COVID-19 study is a prospective observational cohort study conducted entirely online through the Heart Hive, evaluating the impact of the COVID-19 pandemic on patients with cardiomyopathy and subjects without heart disease. All subjects provided electronic informed consent.

Eligibility criteria for the Heart Hive COVID-19 study included subjects aged 18 or over, capacity to provide informed consent and either a self-reported diagnosis of cardiomyopathy, or no history of heart disease. Subjects without heart disease were enrolled as a control group. Exclusion criteria included patients from vulnerable groups (subjects under 18, prisoners, those in a dependent relationship, mental illness) and patients with significant coronary artery disease (cardiac dysfunction attributed to coronary artery disease or prior coronary revascularisation), primary valve disease, congenital heart disease or severe hypertension. Enrolled subjects completed online surveys via the Heart Hive portal. The surveys were adapted from the 'WHO Survey tool and guidance: behavioural insights on COVID-19' (online supplemental appendix 1). ${ }^{10}$ The objectives of the survey were to evaluate the impact of the pandemic, focusing on physical health, the delivery of clinical care, psychological well-being and behavioural adaptations. 


\section{NHS Digital Hospital Episodes Statistics for the NHS of England}

To assess the impact of the COVID-19 pandemic on cardiomyopathy-related hospital admissions, we accessed data from NHS Digital Hospital Episodes Statistics (HES). Corresponding national COVID-19 admission data were acquired from the UK Government website. ${ }^{89}$ To access HES data, a formal data access request was submitted providing scientific justification for the information required. This was approved by the Independent Group Advising on the Release of Data and a data sharing agreement between NHS digital, the investigators and the Trust's Caldicott Guardian was signed prior to the release of data. All available record-level data on hospital admissions specifically due to a primary diagnosis of DCM or HCM, defined by ICD codes I42.0 (DCM), I42.1 (HCM, obstructive) and I42.2 (HCM, other), was obtained in all age groups across England from 2019 to 2020. Data were fully anonymised with each individual patient having a unique HES-ID. Finished consultant episodes were aggregated into spells to represent hospital admissions.

\section{Statistical analysis}

Continuous normally distributed data are expressed as mean $\pm \mathrm{SD}$, and non-normally distributed data as median and IQR. Continuous variables were compared using the Mann-Whitney U test. Categorical data are expressed as number and percentages and compared using $\chi^{2}$ or Fisher's exact test. Statistical analyses were conducted in the SPSS, V.27 (IBM).

\section{Patient and public involvement}

Patients were actively involved in these research initiatives from inception. Both the RBHH CRC Biobank initiative and the Heart Hive platform were designed to address questions raised in consultation with patient focus groups, and patients were involved in the design of both resources. Administrative and patient representatives from the patient charity Cardiomyopathy UK reviewed and contributed to the study design and patient surveys for the Heart Hive COVID-19 study. Following publication, the results of this study will be disseminated to patient members of the charity Cardiomyopathy UK through their magazine and to Heart Hive members through their website (https://www.thehearthive.org/) and social media platforms.

\section{RESULTS}

\section{Royal Brompton \& Harefield Hospital Cardiovascular Research Centre Biobank}

Of the 1683 patients enrolled into the RBHH CRC Biobank, 1236 patients met eligibility criteria (figure 1). This comprised 703 patients with DCM and 533 patients with HCM. There were 840 men $(68.0 \%)$, and the cohort was mostly Caucasian $(\mathrm{n}=996 ; 80.6 \%)$. The mean age was $61.4 \pm 15.6$ years. Patient characteristics are summarised in table 1 .

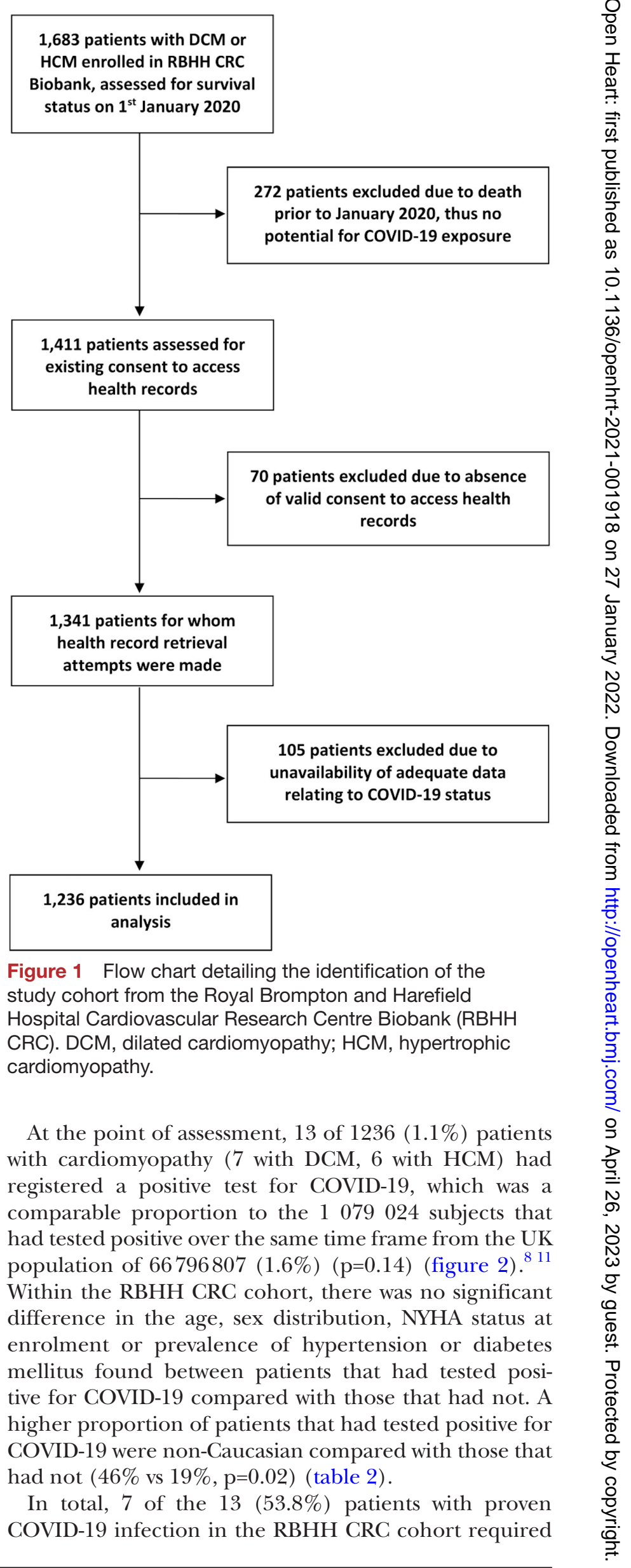




\begin{tabular}{|c|c|c|c|}
\hline & $\begin{array}{l}\text { Total cohort, } \\
n=1236\end{array}$ & $\begin{array}{l}\text { DCM, } \\
n=703\end{array}$ & $\begin{array}{l}\text { HCM, } \\
n=533\end{array}$ \\
\hline Age (years) & $61.4 \pm 15.6$ & $60.8 \pm 15.1$ & $62.2 \pm 16.3$ \\
\hline Male (\%) & $840(68.0)$ & $459(65.3)$ & 381 (71.5) \\
\hline \multicolumn{4}{|c|}{ Self-reported ethnicity (\%) } \\
\hline Afro-Caribbean & $43(3.5)$ & $31(4.4)$ & $12(2.3)$ \\
\hline African & $36(2.9)$ & $20(2.8)$ & $16(3.0)$ \\
\hline Asian & $122(9.9)$ & $40(5.7)$ & $82(15.4)$ \\
\hline Caucasian & $996(80.6)$ & $592(84.2)$ & 403 (75.6) \\
\hline Chinese & $4(0.3)$ & $2(0.3)$ & $2(0.4)$ \\
\hline Mixed & $5(0.4)$ & $2(0.3)$ & $3(0.6)$ \\
\hline Other & $30(2.4)$ & $16(2.2)$ & $15(2.8)$ \\
\hline \multicolumn{4}{|c|}{ NYHA class at enrolment (\%)* } \\
\hline I & $526(45.0)$ & $309(45.6)$ & 217 (44.3) \\
\hline$\|$ & $498(42.6)$ & $273(40.3)$ & 225 (45.9) \\
\hline III & $129(11.0)$ & $87(12.8)$ & $42(8.6)$ \\
\hline IV & $15(1.3)$ & $9(1.3)$ & $6(1.1)$ \\
\hline
\end{tabular}

${ }^{*}$ Missing data, $\mathrm{n}=68,5.5 \%$.

DCM, dilated cardiomyopathy; HCM, hypertrophic

cardiomyopathy; NYHA, New York Heart Association.

hospitalisation during their illness, which was a higher proportion than the $178394(16.5 \%)$ subjects from the UK population that required hospitalisation from 1079024 confirmed COVID-19 cases registered over the same time frame $(\mathrm{p}=0.002){ }^{8}$

Shielding was recommended to 271 of the 1236 (21.9\%) patients with cardiomyopathy in the RBHH CRC Biobank
Table 2 A comparison of patient characteristics between patients with cardiomyopathy that had registered a positive COVID-19 test result and those that had not in the Royal Brompton and Harefield Hospital Cardiovascular Research Centre Biobank

\begin{tabular}{clll}
\hline & $\begin{array}{l}\text { Patients without } \\
\text { positive COVID-19 } \\
\text { test recorded } \\
\text { (n=1223) }\end{array}$ & $\begin{array}{l}\text { Patients } \\
\text { with positive } \\
\text { COVID-19 test } \\
\text { recorded } \\
\text { (n=13) }\end{array}$ & P value \\
\hline Age (years) & $61.4 \pm 15.7$ & $60.5 \pm 10.1$ & 0.54 \\
\hline Male & $830(67.9)$ & $10(76.9)$ & 0.49 \\
\hline Caucasian & $988(80.8)$ & $7(53.8)$ & 0.02 \\
\hline NYHA at enrolment & & \\
\hline I & 523 & 3 & 0.43 \\
II & 490 & 8 & \\
III & 127 & 2 & \\
IV & 15 & 0 & 0.76 \\
\hline Hypertension & $403(33)$ & $5(38.5)$ & 0.79 \\
\hline Diabetes & $123(10.1)$ & $1(7.7)$ & \\
\hline
\end{tabular}

NYHA, New York Heart Association.

(171 with DCM, 100 with HCM). In an unadjusted comparison, this represented a greater proportion than among the UK population $(21.9 \%$ vs $3.9 \%, \mathrm{p}<0.001){ }^{9}$ Overall, there was no difference in the age $(p=0.6)$ or sex distribution $(p=0.6)$ of patients advised to shield among the RBHH CRC Biobank cohort compared with those not advised to shield. However, a higher proportion of those advised to shield had symptoms that markedly or severely limited activity at enrolment (New York Heart Association (NYHA) III or IV) compared with patients that were not advised to shield $(22.2 \%$ vs $9.5 \%, \mathrm{p}<0.001)$.
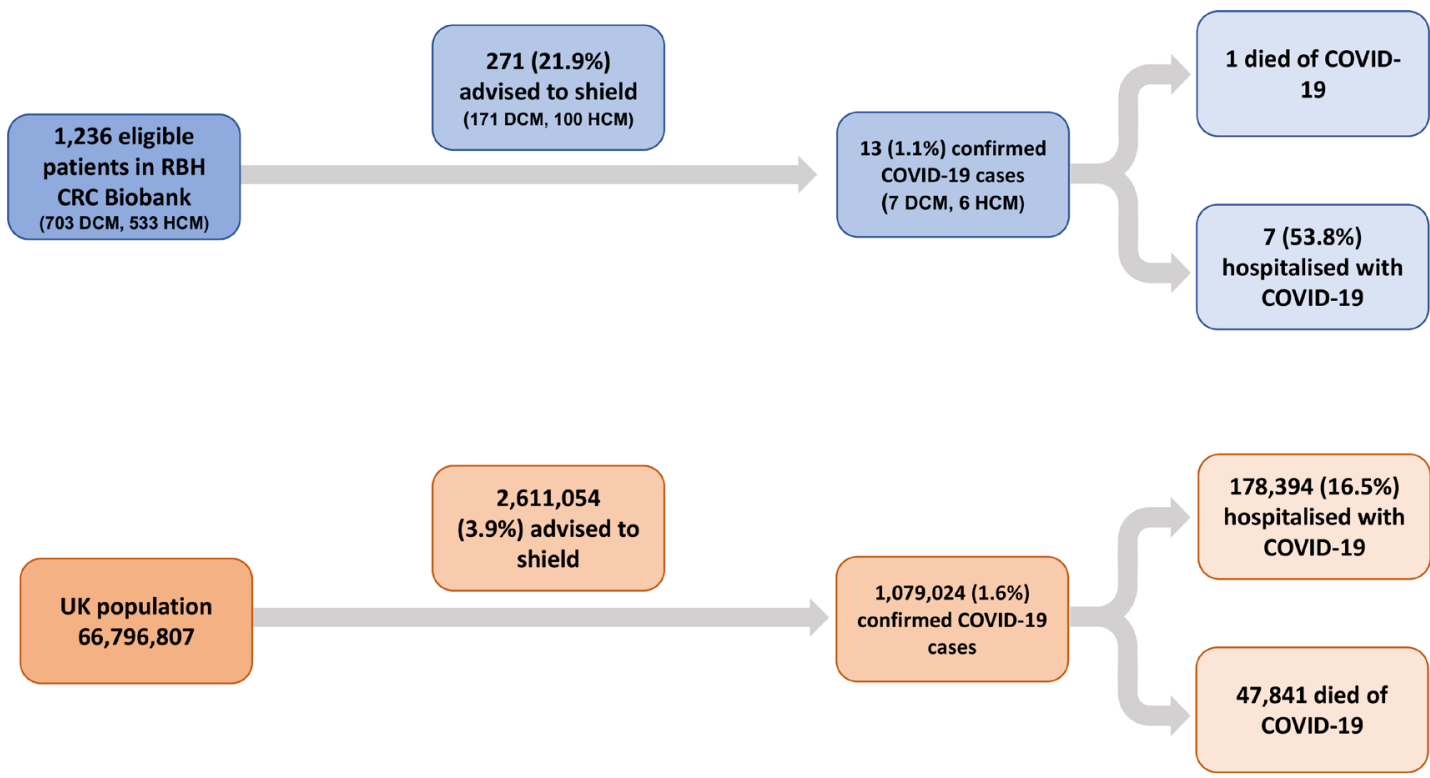

Figure 2 Flow chart illustrating shielding recommendations, positive COVID-19 test results and COVID-19 hospitalisations in the Royal Brompton and Harefield Hospital Cardiovascular Research Centre (RBHH CRC) Biobank cardiomyopathy cohort (in blue) and in the United Kingdom population (in orange). DCM, dilated cardiomyopathy; HCM, hypertrophic cardiomyopathy. 


\begin{tabular}{llll}
\hline $\begin{array}{l}\text { Table } 3 \\
\text { Heart Hive COVID-19 study }\end{array}$ & $\begin{array}{l}\text { Baseline characteristics of subjects enrolled in the } \\
\text { Subjects with } \\
\text { cardiomyopathy } \\
\text { (n=131) }\end{array}$ & $\begin{array}{l}\text { Subjects without } \\
\text { heart disease } \\
\text { (n=76) }\end{array}$ & P value \\
\hline Age & $56.3 \pm 13.4$ & $54.3 \pm 17.7$ & 0.91 \\
Female & $81(61.8)$ & $49(64.5)$ & 0.77 \\
Caucasian & $127(96.9)$ & $68(89)$ & 0.17 \\
Diagnosis & & & \\
DCM & $65(49.6)$ & N/A & \\
HCM & $46(35.1)$ & & \\
ACM & $10(7.6)$ & & \\
$\begin{array}{l}\text { Other } \\
\text { cardiomyopathies }\end{array}$ & $10(7.6)$ & & \\
\hline
\end{tabular}

ACM, arrhythmogenic cardiomyopathy; DCM, dilated cardiomyopathy; HCM, hypertrophic cardiomyopathy; N/A, not applicable.

Proportionally more patients with DCM were advised to shield than patients with HCM (24.3\% vs $18.8 \%$, $\mathrm{p}=0.02$ ). Patients with DCM that were advised to shield had a lower baseline left ventricular ejection fraction $(35 \%$ (26.5-47) vs $43.5 \%$ (31-52), $\mathrm{p}<0.001$ ), a higher proportion with NYHA III or IV symptoms at enrolment $(24.3 \%$ vs $12.1 \%$, $\mathrm{p}<0.001)$ and a trend towards a higher proportion with fibrosis identified on CMR ( $42.1 \%$ vs $32.7 \%, \mathrm{p}=0.05)$ compared with those not advised to shield. Of the 271 patients with cardiomyopathy that had been advised to shield, $4(1.5 \%)$ had had a positive COVID-19 test at the point of evaluation, compared with 9 of the $965(0.9 \%)$ patients that were not advised to shield.

\section{The Heart Hive COVID-19 study}

The Heart Hive COVID-19 study population comprised 207 subjects who enrolled between 22 July 2020 and 18 November 2020 . There were 130 women $(63.8 \%)$, and the majority of the cohort was Caucasian $(n=195,94.2 \%)$. The mean age was $55.6 \pm 15.1$ years. Within this cohort, 131 had a self-reported diagnosis of cardiomyopathy and the remaining 76 had no known heart disease. There was no significant difference in the sex distribution, ethnicity or age between patients with cardiomyopathy and subjects without heart disease. The cardiomyopathy group comprised 65 patients with DCM, 46 patients with HCM, 10 patients with arrhythmogenic cardiomyopathy and 10 individuals with other cardiomyopathies. The baseline characteristics of the cohort are shown in table 3 .

\section{The impact of the pandemic on physical health}

A higher proportion of patients with cardiomyopathy reported that they had experienced a subjective deterioration in physical health occurring as a result of the pandemic compared with the subjects without heart disease $(31.3 \%$ vs $13.2 \%, p=0.004)$. Among the patients with cardiomyopathy, the number of patients experiencing this subjective deterioration in physical health significantly exceeded the proportion that had reported a suspected COVID-19 infection (6 patients $(4.6 \%)$ with potential symptoms of COVID-19 but none with confirmatory tests) (figure 3).

\section{The availability and delivery of clinical care during the pandemic}

Of the 131 patients with cardiomyopathy, 38 (29.0\%) reported rescheduled outpatient clinic visits, 21 (16.0\%) reported a cancelled clinic appointment without rescheduling, $51(38.9 \%)$ reported having missed clinical investigations, $10(7.6 \%)$ reported having procedures cancelled or postponed and $7(5.3 \%)$ reported missing doses of medication due to the pandemic. Importantly, 53 patients with cardiomyopathy $(40.4 \%)$ felt their ongoing health needs could not be adequately met by telemedicine (figure 3).

\section{The psychological impact of the pandemic}

Scores assigned by study participants representing their level of fear $(\mathrm{p}=0.24)$, stress $(\mathrm{p}=0.45)$, worry $(\mathrm{p}=0.37)$, helplessness $(\mathrm{p}=0.73)$ and depression $(\mathrm{p}=0.57)$ associated with the pandemic, and the degree to which they felt their thoughts were occupied by the pandemic $(p=0.29)$, did not differ between patients with cardiomyopathy and subjects without heart disease (figure 4).

\section{Behavioural adaptations}

A higher proportion of patients with cardiomyopathy had received a recommendation to shield compared with the subjects without heart disease $(21.4 \%$ vs $9.2 \%, \mathrm{p}=0.02)$. Of the 28 patients with cardiomyopathy that had received a recommendation to shield, in 20 patients $(71.4 \%)$ this was due to their underlying cardiomyopathy, in 2 patients $(7.1 \%)$ it was due to cardiomyopathy plus a least one additional reason and in 6 patients $(21.4 \%)$ due to an alternative reason unrelated to cardiomyopathy.

An important proportion of the whole cohort (28.5\%) reported that they would either not attend hospital, or would be very reluctant to attend hospital, in the event that they felt extremely unwell during the pandemic. Patients with cardiomyopathy were no less likely to attend hospital under such circumstances than subjects without heart disease $(26.7 \%$ vs $31.6 \%, \mathrm{p}=0.52)$. Patients with cardiomyopathy felt they were more susceptible to COVID-19 infection $(p<0.001)$ and that they would suffer a more severe illness if infected $(\mathrm{p}<0.001)$ compared with subjects without heart disease. Among the patients with cardiomyopathy, $16.8 \%$ reported that they would either refuse to attend or be very reluctant to attend hospital if symptoms from their cardiomyopathy deteriorated, although only $61.1 \%$ of the patients with cardiomyopathy felt able to distinguish symptoms of COVID-19 from symptoms related to their underlying heart condition.

\section{Patients with cardiomyopathy that had received a recommendation to shield}

A comparable proportion of patients with cardiomyopathy that were advised to shield reported a subjective deterioration in their physical health compared with those that were not advised to shield $(39.3 \%$ vs $29.1 \%$, 


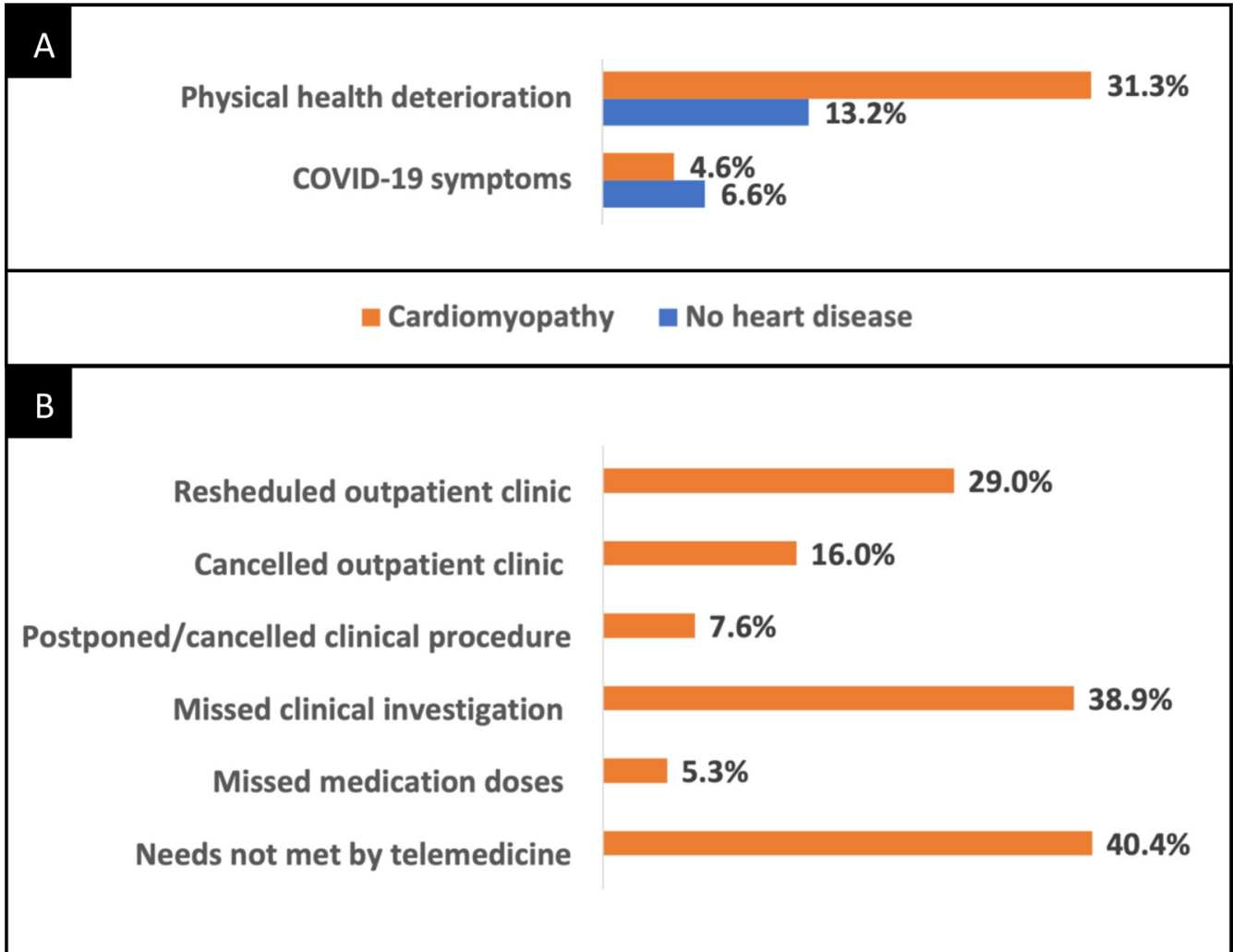

Figure 3 Reported changes in physical health for patients with cardiomyopathy and subjects without heart disease in the Heart Hive COVID-19 study demonstrating that significantly more patients with cardiomyopathy experienced subjective deterioration in physical health. $(A)$ and the reported provision of healthcare for patients with cardiomyopathy during the pandemic $(B)$.

$\mathrm{p}=0.36$ ); however, proportionally more of those advised to shield reported worsening of symptoms related to their cardiomyopathy $(21.4 \%$ vs $6.8 \%, \mathrm{p}=0.03)$. There were no significant differences in the psychological measures assessed between patients based on whether they had been advised to shield or not.

\section{NHS Digital HES for the National Health Service of England}

There were 5161 hospital admissions due to DCM or HCM across all ages in England in 2020, compared with 6286 admissions in 2019 (17.9\% year-on-year reduction). Individually, there was a $16.5 \%$ reduction in admissions due to DCM (4398 in 2020 vs 5266 in 2019) and a $25.2 \%$ reduction in admissions due to HCM (763 in
2020 vs 1020 in 2019). There was heightened disparity in cardiomyopathy-related hospital admissions during the first UK national lockdown (23 March 2020-19 June 2020 ), with a $40.1 \%$ reduction in hospital admissions due to DCM, and a $51.6 \%$ reduction in admissions due to HCM, compared with the corresponding time period in 2019. The reduction in cardiomyopathy-related hospital admissions was less marked after the first UK National lockdown was lifted and before the second national lockdown began (20 June 2020-4 November 2020), during which period there was a $7.4 \%$ reduction in DCM-related hospital admissions and a $23.0 \%$ reduction in $\mathrm{HCM}$ admissions compared with the corresponding time period

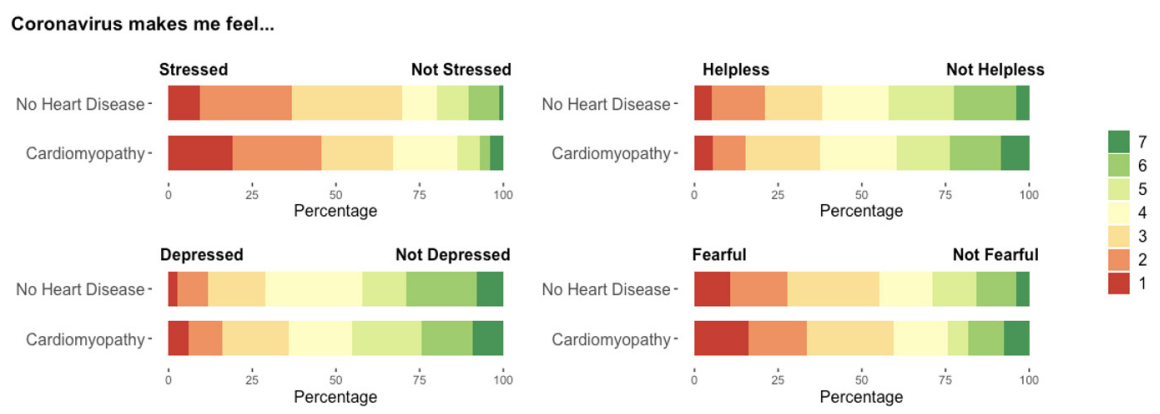

Figure 4 The psychological impact of the COVID-19 pandemic did not differ between patients with cardiomyopathy and subjects without heart disease enrolled in the Heart Hive COVID-19 study: results from survey questions related to patient perception of psychological measures during the pandemic. 


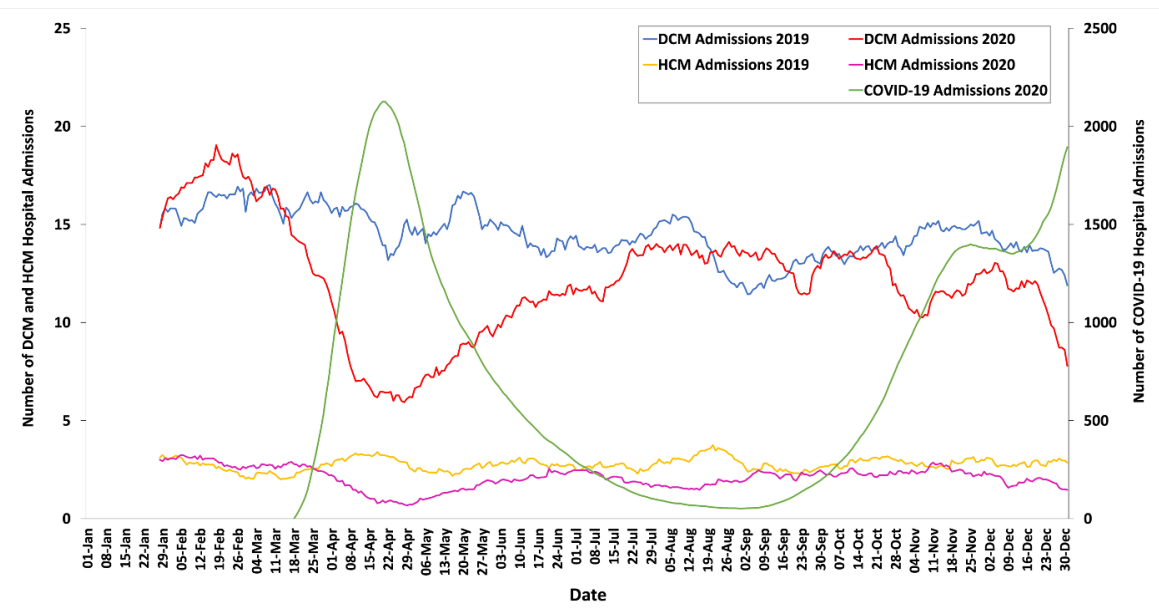

Figure 5 The impact of the COVID-19 pandemic on hospital admissions with a primary diagnosis of dilated cardiomyopathy or hypertrophic cardiomyopathy across NHS England, 2019-2020. Hospital admission data are presented as 28-day moving averages. DCM, dilated cardiomyopathy; HCM, hypertrophic cardiomyopathy; NHS, National Health Service.

in 2019. During the second UK lockdown (5 November 2020-2 December 2020), there was a $12.9 \%$ reduction in DCM-related hospital admissions and a $25.6 \%$ reduction in HCM admissions compared with the same period in 2019 (figure 5). The age of patients admitted to hospital due to cardiomyopathy did not differ significantly between 2019 and 2020 (median age 58, IQR 47-70 vs median age 59, IQR 46.25-75; $\mathrm{p}=0.52$ ). Similarly, there was no significant difference in the sex distribution of the patients admitted to hospital due to cardiomyopathy between 2019 and 2020 (65.7\% male in 2019 vs $64.7 \%$ in 2020; $\mathrm{p}=0.27$ ).

\section{DISCUSSION}

Leveraging three parallel research initiatives, we draw important insight into both the direct and indirect impact of the COVID-19 pandemic on patients with cardiomyopathy. Our findings from the RBHH CRC Biobank indicate that patients with cardiomyopathy do not appear to be at greater risk of testing positive for COVID-19 than the general population. In our unadjusted analysis, a higher proportion of the patients with cardiomyopathy that had registered a positive COVID-19 test also required treatment in hospital compared with the background UK population during the early stages of the pandemic. Across both the RBHH CRC biobank and the Heart Hive COVID-19 study cohorts, approximately one in five patients with cardiomyopathy received a recommendation to shield (21.9\% in RBHH CRC Biobank; $21.4 \%$ in Heart Hive COVID-19 study) at the discretion of their doctors. Our findings from the RBHH CRC Biobank indicate that their assessment of risk was broadly in line with conventional markers of disease severity. In the Heart Hive COVID-19 study, nearly a third of surveyed patients with cardiomyopathy experienced subjective deterioration in their physical health during the pandemic despite a much lower proportion reporting possible COVID-19 symptoms, suggesting a significant contribution of the indirect effects of the pandemic. We illustrate how these indirect effects span multiple components of routine care. We found there to be no significant difference in the impact of the pandemic on the psychological measures assessed between patients with cardiomyopathy and subjects without heart disease, perhaps reflecting the broader psychological impact of the pandemic on the population. From the NHS Digital HES dataset, we demonstrate a significant year-on-year reduction in DCM and HCM related hospital admissions in England between 2019 and 2020, with the most marked reduction coinciding with the first national lockdown.

A strength of our research initiative lies in the multiscale approach. The observed decline in cardiomyopathyrelated admissions from NHS Digital HES dataset is afforded plausibility when considered in tandem with behavioural insights from the Heart Hive COVID-19 study, which demonstrated that only a modest proportion of patients with cardiomyopathy would attend hospital if they felt extremely unwell during the pandemic. A potential weakness in the RBHH CRC Biobank initiative lies in varied access to widespread testing for COVID-19 in the early phase of the pandemic, thus, the authors cannot exclude a selection bias occurring due to preferential testing of more unwell patients that were more likely to require hospital care. The authors also recognise the low proportion of patients that had registered a positive test in the RBHH CRC cohort at the point of assessment and cannot exclude this small sample size conflating the described association of a higher rate of hospitalisation among those with cardiomyopathy who were infected with COVID-19 during the early stages of the pandemic.

A recent study reported a reduction in heart failure admission rates during the early stages of the pandemic compared with preceding hospitalisation rates. ${ }^{12}$ This trend has been observed across a spectrum of cardiovascular conditions leading many to question whether it reflects a reduction in disease incidence or simply 
reluctance to attend hospital ${ }^{12}{ }^{13}$; the behavioural insights gained from the Heart Hive COVID-19 study implicates the latter as a significant contributor to this phenomenon. A further large study of patients with established cardiovascular disease has identified lower overall in-hospital mortality and higher out-of-hospital mortality during a period of COVID-19 lockdown compared with data preceding the pandemic. ${ }^{14}$ Our research initiatives contextualise this observation, demonstrating marked interruptions to routine care, an unwillingness to seek emergency care among many patients, and a reduction in cardiomyopathy-related hospital admissions.

One potential explanation for the finding of a higher rate of COVID-19 hospitalisation among patients with cardiomyopathy in the RBHH CRC cohort compared with the UK population could be that patients with cardiomyopathy are at higher risk of more severe illness from COVID-19. Recommendations to shield in the UK were largely made before such data on disease-specific COVID-19 susceptibility and the risk of severe illness were available. The harms of shielding are not well characterised, but shielding advice may have exacerbated interruptions to usual care. Studies such as ours that provide insight into the risk of specific patient groups may be important in guiding future recommendations in the event of further waves of infection. However, it is important to note that a greater proportion of those hospitalised for COVID-19 in this cohort were nonCaucasian, which has been identified as a risk factor for more severe disease. ${ }^{15}$

The impact of the COVID-19 pandemic on mortality for patients with cardiomyopathy remains unclear and requires further interrogation with longitudinal outcome data. Whether the reduction in routine and emergency care and the decline in cardiomyopathy-related hospitalisation has resulted in more community cardiovascular deaths and higher symptom burden requires further evaluation. Importantly, the research initiatives presented depict the behavioural patterns of subjects up until the advent of large-scale vaccination. The attitude to COVID-19 immunisation among patients with cardiomyopathy and potential changes in perception of risk will be examined in follow-up reports from the Heart Hive COVID-19 Study. Finally, although not powered to detect the effect of shielding on the rate of infection in the initiatives presented, no absolute reduction in infections was demonstrated among those advised to shield in the RBHH CRC cohort. Larger studies evaluating the efficacy of shielding in reducing COVID-19 infections in specific patient groups are eagerly awaited, especially in light of findings from the Heart Hive COVID-19 study that a higher proportion of patients with cardiomyopathy advised to shield reported a deterioration in cardiomyopathy-related symptoms.

We conclude that patients with cardiomyopathy represent a complex and varied group in whom the impact of the pandemic reaches beyond simply those that have been infected with COVID-19. The scale remains undetermined, yet the practical relevance of our findings span other patient populations across different medical disciplines, especially those with complex health needs, and should be considered amidst plans to reorganise healthcare services as the pandemic evolves.

\section{Author affiliations}

${ }^{1}$ National Heart and Lung Institute, Imperial College London, London, UK

${ }^{2}$ Cardiovascular Research Centre, Royal Brompton and Harefield Clinical Group,

Guy's and St Thomas' NHS Foundation Trust, London, UK

${ }^{3}$ MRC London Institute of Medical Sciences, Imperial College, London, UK

${ }^{4}$ Department of Epidemiology and Biostatistics, Imperial College London School of Public Health, London, UK

${ }^{5}$ British Heart Foundation Centre of Excellence, Imperial College London, London, UK

${ }^{6}$ Department of Hygiene and Epidemiology, University of loannina, Ioannina, Greece

Acknowledgements The authors thank the research team at the Royal Brompton and Harefield Hospital Cardiovascular Research Centre, in particular Geraldine Sloane and the research nurses. The research team additionally thank the study participants and the supporters of the Heart Hive community.

Contributors Conception and study design: DJH, RJB, ASL, LM, AMR, SKP and JSW. Data acquisition, analysis or interpretation: DJH, RJB, ASL, LM, REJ, BPH, UT, DM, AD, IT, AJB, AP, AMR, SKP and JSW. Drafting of manuscript: DJH, JSW, SKP, ASL, LM, DM and AD. Critical revision of manuscript for important intellectual content: RJB, ASL, LM, REJ, BPH, UT, IT, AJB, AP, AMR, SKP and JSW. Final approval of manuscript: DJH, RJB, ASL, LM, REJ, BPH, UT, DM, AD, IT, AJB, AP, AMR, SKP and JSW. Overall responsibility for the content as guarantors: DJH and JSW.

Funding This work was supported by the Wellcome Trust (107469/Z/15/Z), Medical Research Council (UK), British Heart Foundation (RE/18/4/34215), National Institute for Health Research (NIHR) Royal Brompton and Harefield Cardiovascular Biomedical Research Unit, the NIHR Imperial College Biomedical Research Centre, Sam's Rainbow Foundation and Cardiomyopathy, UK

Competing interests JSW has acted as a consultant for MyoKardia and Foresite Labs (unrelated to this work).

Patient consent for publication Not applicable.

Ethics approval This study was approved by (1) Cardiovascular Research Centre Biobank: South Central-Hampshire B Research Ethics Committee, REC Reference 09/H0504/104+5. (2) Heart Hive COVID-19 Study: London-Fulham Research Ethics Committee, REC Reference 20/HRA/2744.

Provenance and peer review Not commissioned; externally peer reviewed.

Data availability statement All data relevant to the study are included in the article or uploaded as online supplemental information.

Open access This is an open access article distributed in accordance with the Creative Commons Attribution Non Commercial (CC BY-NC 4.0) license, which permits others to distribute, remix, adapt, build upon this work non-commercially, and license their derivative works on different terms, provided the original work is properly cited, appropriate credit is given, any changes made indicated, and the use is non-commercial. See: http://creativecommons.org/licenses/by-nc/4.0/.

\section{ORCID iD}

Daniel J Hammersley http://orcid.org/0000-0002-1164-5993

\section{REFERENCES}

1 Wu Z, McGoogan JM. Characteristics of and Important Lessons from the Coronavirus Disease 2019 (COVID-19) Outbreak in China: Summary of a Report of 72314 Cases from the Chinese Center for Disease Control and Prevention. JAMA 2020;323:1239-42.

2 Ssentongo P, Ssentongo AE, Heilbrunn ES, et al. Association of cardiovascular disease and 10 other pre-existing comorbidities with COVID-19 mortality: a systematic review and meta-analysis. PLoS One 2020;15:e0238215.

3 Ball S, Banerjee A, Berry C, et al. Monitoring indirect impact of COVID-19 pandemic on services for cardiovascular diseases in the UK. Heart 2020;106:1890-7.

4 Maron BJ, Towbin JA, Thiene G, et al. Contemporary definitions and classification of the cardiomyopathies: an American Heart 
Association scientific statement from the Council on Clinical Cardiology, Heart Failure and Transplantation Committee; quality of care and outcomes research and functional genomics and translational biology interdisciplinary working groups; and Council on epidemiology and prevention. Circulation 2006;113:1807-16.

5 Authors/Task Force members, Elliott PM, Anastasakis A, et al. 2014 ESC guidelines on diagnosis and management of hypertrophic cardiomyopathy: the task force for the diagnosis and management of hypertrophic cardiomyopathy of the European Society of cardiology (ESC). Eur Heart J 2014;35:2733-79.

6 Pinto YM, Elliott PM, Arbustini E, et al. Proposal for a revised definition of dilated cardiomyopathy, hypokinetic non-dilated cardiomyopathy, and its implications for clinical practice: a position statement of the ESC Working group on myocardial and pericardial diseases. Eur Heart J 2016;37:1850-8.

7 Guidance on shielding and protecting people who are clinically extremely vulnerable from COVID-19 - GOV.UK. Available: https:// www.gov.uk/government/publications/guidance-on-shielding-andprotecting-extremely-vulnerable-persons-from-covid-19/guidanceon-shielding-and-protecting-extremely-vulnerable-persons-fromcovid-19 [Accessed 29 Mar 2021].

8 Daily summary | Coronavirus in the UK. Available: https:// coronavirus.data.gov.uk/ [Accessed 24 Mar 2021].

9 Coronavirus Shielded Patient List open data set, England - NHS Digital. Available: https://digital.nhs.uk/dashboards/shielded-patientlist-open-data-set [Accessed 24 Mar 2021].
10 Survey tool and guidance: behavioural insights on COVID-19, 29 July 2020 (produced by the WHO European region). Available: https://www.euro.who.int/en/health-topics/health-emergencies/ coronavirus-covid-19/publications-and-technical-guidance/ risk-communication-and-community-engagement/who-tool-forbehavioural-insights-on-covid-19/survey-tool-and-guidancebehavioural-insights-on-covid-19-produced-by-the-who-europeanregion [Accessed 29 Mar 2021].

11 Overview of the UK population - Office for National Statistics. Available: https://www.ons.gov.uk/peoplepopulationandcommunity/ populationandmigration/populationestimates/articles/overviewofth eukpopulation/january2021 [Accessed 28 Mar 2021].

12 Bromage DI, Cannatà A, Rind IA, et al. The impact of COVID-19 on heart failure hospitalization and management: report from a heart failure unit in London during the peak of the pandemic. Eur $\mathrm{J}$ Heart Fail 2020;22:978-84.

13 Metzler B, Siostrzonek P, Binder RK, et al. Decline of acute coronary syndrome admissions in Austria since the outbreak of COVID-19: the pandemic response causes cardiac collateral damage. Eur Heart $J$ 2020;41:1852-3.

14 Butt JH, Fosbøl EL, Gerds TA, et al. All-cause mortality and location of death in patients with established cardiovascular disease before, during, and after the COVID-19 lockdown: a Danish nationwide cohort study. Eur Heart J 2021;42:1516-23.

15 Lassale C, Gaye B, Hamer M. Ethnic disparities in hospitalization for COVID-19: a community-based cohort study in the UK. medRxiv Prepr Serv Heal Sci. 